Page | 9

\title{
The Determinants of Cloud Computing Adoption in Healthcare
}

\section{Ahmad AlTwaijiry}

\section{How to cite:}

AlTwaijiry, A. (2020) 'The Determinants of Cloud Computing Adoption in Healthcare', ResearchBerg Review of Science and Technology, 1(1), pp. 9-20.

Article history:

Received: 2020/03/21

Available online: $2020 / 08 / 11$

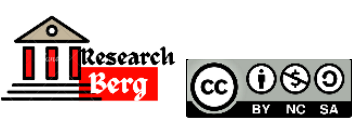

This work is licensed under a Creative Commons Attribution-NonCommercial 4.0 International License.

\section{Abstract}

Cloud computing is useful for the healthcare sector since it reduces complexity, enables efficient administration, and facilitates collaboration between the systems in healthcare sectors. This research seeks to examine the factors affecting the adoption of cloud computing in healthcare. It used three robust least square estimation techniques such as S-estimation, M-estimation, and MM-estimation. The findings suggest that the determinants of adoption of cloud computing are similar to other business institutions such as compatibility, technological preparedness, complexity, security, competitive constraints, savings on costs, assistance to senior management, vendor assistance.

\section{Keywords: Cloud computing adoption, Healthcare, Robust Least square}

\section{Introduction}

The majority of current health care systems are based on processes that include paper medical records, repeated test results, non-digitized photos, handwritten notes, fragmented information technology systems, and siloed data (Abiy et al., 2018). Data exchange between providers is inefficient, and data portability is uncommon. Consumers and patients lack transparency on health plan fees and covered treatments, and health insurance web sites provide few information. Physicians continue to communicate mostly via pagers, and organizing care plans and other administrative operations remains inefficient. 
Cloud computing is being heralded as the preeminent answer to the challenges of unpredictable traffic surges, computer overloads, and potentially costly expenditures in data processing and backup gear (Avram, 2014). It has the potential to disrupt the IT sector, increasing the attractiveness of software and infrastructure as services by altering how hardware is developed and acquired. Cloud computing is a computing paradigm that complements the present consumption

Page | 10 and delivery model for Internet-based IT services by enabling dynamically scaled and often virtualized resources to be shared over the internet (ogly Alguliev and ogly Alekperov, 2013).

The cloud's on-demand computing capability offers value, particularly when healthcare institutions and providers need rapid deployment, access, and management of network information. With the increased need for data-based security, there has to be a paradigm change in the development, use, improved storage, cooperation, and sharing of healthcare data solutions (Sultan, 2014). This is where cloud computing excels.

Healthcare is one such area that has been a leader in cloud adoption. Across the world, healthcare providers are realizing the full potential of cloud technologies.

The use of cloud computing in the healthcare business began years ago, but progressed slowly due to several obstacles. It has received fresh speed in recent years as a result of technological advancements and higher client demands. Thus, moving to the cloud is no longer a choice for healthcare organizations; it is a need dictated by the new reality (Doukas and Maglogiannis, 2012).

The healthcare sector is difficult due to the breadth of its procedures and the volume of private and sensitive information it must manage. The complexity of the sector often results in two significant challenges: increasing operating costs (including data storage costs) and difficulties developing a self-sufficient health environment. Historically, technology has been the rescuer, providing a workaround for key healthcare business difficulties. Cloud computing is one such technology. It has been used in the healthcare business for some years and has evolved in response to industry developments. Cloud computing is altering the healthcare business on a variety of levels due to its collaborative, scalable, reachable, efficient, and secure capabilities.

Prior to making any technology selection choice, speed is a critical factor to consider. Cloud-based solutions can update and improve its capabilities at an impressive rate with no interaction, and one can also get real-time updates on any pertinent information.

Cloud computing's advantages in healthcare include unequaled speed and increased access to information, which may help overcome roadblocks encountered by industry stakeholders and patients (Dang et al., 2019). This future technology has also altered the scope of clinical research, and the cloud may be used to manage clinical trials and share information.

Organizations in the healthcare sector work in an ever-changing environment. The cloud enables healthcare technology such as electronic medical records, mobile applications, patient portals, IoT devices, and big data analytics (Shimrat, 2009). It enables effortless scalability and flexibility, which ultimately enhances the decision-making process. 
Along with maintaining $24 \times 7$ availability, healthcare providers must significantly increase their data storage and network needs to meet service expectations. Cloud technology enables healthcare practitioners to scale up or down their storage requirements as needed.

Due to the fact that cloud migration may fundamentally disrupt healthcare providers' standard

Page | 11 data management processes, healthcare providers must plan for the migration process well in advance (Rahimi, Møller and Hvam, 2016). Not only does a cloud migration plan mitigate risks, but it also decreases the likelihood of downtime, eliminates information breaches, enhances data management, and fortifies security standards.

\section{Literature review.}

Several studies have examined the adoption of cloud commuting technology in healthcare. The aspects that will affect the adoption of Cloud Computing in Saudi healthcare organizations are examined and identified by Alharbi, Atkins and Stanier, (2016). To provide a holistic evaluation of the determinants of Cloud Computing adoption in healthcare organizations, the study integrates the TOE (Technology-Organization-Environment) framework with the Information System Strategic Triangle (IS Triangle) and the HOT-fit (Human-Organization-Technology) model. The Business viewpoint was judged to be the most essential of the five views evaluated in this research, followed by the Technology, Organizational, and Environmental perspectives, and lastly the Human perspective. Soft financial analysis, relative advantage, hard financial analysis, attitude toward change, and pressure from partners in the business ecosystem are the five most significant elements impacting Cloud Computing adoption in this context, according to the study's results.

Sulaiman and Magaireah, (2014) used the TOE framework to investigate the variables that influence the adoption of an integrated cloud-based e-health record EHR system in Jordanian healthcare institutions. Interviews with IT specialists at Jordanian healthcare organizations are used to perform the research. Technological context "privacy, security, and reliability," organizational context "top management support, and technological readiness," and environmental context "government policy, legal environment, and competition" are the three TOE contexts that influence cloud-based EHR adoption.

Mallmann and Maçada, (2018) investigated the variables that influence cloud computing (CC) adoption in public and private hospitals in developing countries, especially in Brazil's hospital business. Authors spoke with IT specialists from four different hospitals. The report shows that hospitals are more concerned about security and availability than the predicted advantages of CC, such as flexibility and cost savings. Furthermore, the data demonstrate that there are significant distinctions between public and private enterprises, such as regulatory constraints and cost, that impact cloud computing adoption in each environment. Companies that deal with sensitive data, such as healthcare institutions, continue to be concerned about cloud-based services. The information gained from this research will be especially useful to IT leaders as they make decisions on whether or not to implement or amend CC projects in healthcare.

The Technological Acceptance Model, the Technology-Organization-Environment Model, and the Theory of Planned Behavior were all combined in the research by Ogwel, Otieno and Odhiambo, (2020) to give a deeper theoretical lens for examining variables impacting cloud 
adoption. The purpose of this research was to ascertain the effect that technical, organizational, and behavioral settings have on cloud computing adoption. The study's target audience was 114 healthcare professionals (facility administrators and health records and information officers) working in public hospitals. The research used a cross-sectional survey to gather data on a single day, utilizing questionnaires sent to randomly selected employees. The connection and intensity of

Page | 12 the association between the anticipated variables and cloud computing adoption were determined using logistic regression. The incidence of cloud computing use was 58 percent among public hospitals. In terms of cloud computing service models, $100 \%$ of users used Software-as-a-Service, followed by $5 \%$ who used Platform-as-a-Service and none who used Infrastructure-as-a-Service. The research discovered that cloud computing adoption is significantly influenced by technology readiness, service quality, expert scarcity, top management support, company size, perceived utility, perceived simplicity of use, and social impact. The results of this research will aid healthcare organizations in better understanding the factors that influence cloud computing adoption and will assist them through the process of adoption. Cloud computing vendors may also utilize the results of this study to address areas of concern, therefore gaining the trust of Kenyan healthcare organizations.

Ratnam and Dominic, (2014) examines the interactions between healthcare providers, information technology resources, and health insurance providers in order to determine cloud computing adoption. The findings of this research indicate that information technology resources have a considerable impact on healthcare providers and health insurance providers. With the implementation of the cloud, efficiency and healthcare services may be improved. The conclusions of this research include reliability analysis, descriptive statistics, factor loading, regression, and correlations for IT capabilities in healthcare services. Additionally, structural equation modeling is utilized to determine the overall model fit. Modeling structural equations (SEM)

\section{Variables}

\subsection{Dependent variable}

\subsubsection{Cloud Computing Adoption}

Cloud Computing Adoption refers to the goal of a healthcare institution to embrace cloud computing services.

A) Rather than stop, my institution wants to continue utilizing our cloud computing products.

B) Rather than employing an alternative method, I intend to continue using our cloud computing service (traditional software).

C) If possible, I would prefer to cancel my subscription to our cloud computing service.

\subsection{Independent variables}

\subsubsection{Compatibility}

Compatibility refers to the degree to which the value of cloud computing aligns with an adopter's current values, beliefs, and requirements. 
Page | 13

A) Ongoing cloud computing usage will be compatible with all areas of my institution's operations.

B) The institution's continued usage of cloud computing is consistent with the way I like to work.

C) Continuous cloud computing usage is fully consistent with my present job responsibilities at the school.

D) Cloud computing is simple to connect with our current systems.

E) Cloud computing is culturally and ethically acceptable.

\subsubsection{Technological Preparedness}

The term "Technology Readiness" refers to the technical capabilities of an organization, such as the availability of IT personnel and the IT infrastructure.
A) My institution is aware of the benefits of cloud computing in supporting our operations.
B) My institution's IT infrastructure is capable of supporting ongoing cloud computing usage.
C) My institution is committed to educating consumers about cloud computing.
D) My organization is well-versed in cloud computing.

\subsubsection{Complexity}

Complexity refers to the degree of difficulty associated with comprehending, using, or continuing to use cloud computing.
A) Continuous cloud computing use requires considerable mental effort.
B) Continuous cloud computing use is annoying.
C) Continuous cloud computing use is just too complicated.
D) The abilities required to continue utilizing cloud computing are incomprehensible to the average user.

\subsubsection{Security}

A) Security refers to the degree to which cloud computing meets the security needs of HEIs.

B) When I use cloud computing solutions, the confidentiality and security of my institution's data are guaranteed.

C) In the event of harm, current liability legislation makes it quite obvious who will take responsibility.

D) My organization will not be harmed by the cloud computing service provider using contractual gaps.

E) Cloud computing ensures the security of the institution's data.

F) Cloud computing systems will ensure that the institution's data is adequately protected. 
G) Cloud computing companies have enhanced security measures in place to protect the institution's data.

\subsubsection{Competitive Constraints}

Competitive Pressure (CP) is the perception by executives of an institution that rivals have gained

Page | 14 a significant competitive advantage by using cloud computing services (for example, in terms of teaching and learning effectiveness).

A) Cloud computing is being used by an increasing number of institutions to perform educational activities and communication.

B) Cloud computing is being used by an increasing number of institutions to manage and share information.

C) Cloud computing is being used by an increasing number of institutions to manage projects and learning.

\subsubsection{Savings on costs}

A) Cloud computing enables innovation, lowers infrastructure expenses, reduces energy usage, and saves maintenance costs.

B) Cloud computing is a more efficient method of computing than the alternative.

C) Cloud computing enables significant time and effort savings.

D) By using cloud computing on a constant basis, institutions may save needless costs and time.

\subsubsection{Assistance to Senior Management}

The vision, support, and commitment to fostering the ideal environment for further cloud computing use in HEIs

A) Top management is likely to incur risks associated with further cloud computing adoption.

B) Top management actively engages in defining a vision for cloud computing and devising plans for its continued usage.

C) Top management expresses their support for further cloud computing adoption.

\section{Vendor Assistance (VS)}

A) Refers to provider operations that have a substantial impact on the likelihood of continuing to use cloud computing.

B) Cloud computing is extensively promoted by vendors.

C) The vendor guarantees a service level agreement.

D) Vendors offer proper technical support for cloud computing.

E) During deployment, cloud computing companies provide easy access to support.

F) Vendors give proper training for cloud computing. 
Questionaire have been adapted from (Qasem et al., 2020). The data has been collected from 280 IT-persons of different healthcare centers.

\section{Page | 15 3. Research Hypotheses}

This research tested the following hypotheses:

H1: Compatibility impacts the adoption of cloud computing

H2: Technological Preparedness impacts the adoption of cloud computing

H3: Complexity impacts the adoption of cloud computing

H4: Security impacts the adoption of cloud computing

H5: Competitive Constraints impacts the adoption of cloud computing

H6: Savings on costs impacts the adoption of cloud computing

H7: Assistance to Senior Management impacts the adoption of cloud computing

H8: Vendor Assistance (VS) impacts the adoption of cloud computing

\section{Estimation techniques}

Ordinary estimators are sensitive to the existence of outlier observations for the regression model under consideration. Because conventional regression techniques are very sensitive to these outlier observations, they may provide coefficient estimates that may not adequately reflect the underlying statistical connection.

Robust least squares is a term that relates to a range of regression techniques that are supposed to be less sensitive to outliers. There are three techniques for computing resilient least squares: Mestimation, S-estimation, and MM-estimation (Faulina, no date). The three approaches vary in their emphasis:

-M-estimation is used to deal with dependent variable outliers, which are values that deviate significantly from the regression model norm (large residuals). If linearity, homoscedasticity, and uncorrelated errors are assumed, the maximum likelihood estimator of is just the OLS estimator obtained by minimizing the sum of squares function.

$$
\min \sum_{i=1}^{n}\left(y_{i}-\sum x_{i j} \beta_{j}\right)^{2}=\min \sum_{i=1}^{n}\left(e_{i}\right)^{2} .
$$


-S-estimation is a time-consuming computational approach that focuses on outliers in the regressor variables (high leverages). It has been recommended examining the magnitude of the residuals in response to the low breakdown point of M-estimators. S-estimates was presented in this vein. S-estimates are the solution that produces the least dispersion of the residuals feasible.

Page | 16

$$
\min \hat{\sigma}\left(e_{1}(\hat{\beta}), \ldots e_{n}(\hat{\beta})\right)
$$

-MM-estimation is a hybrid of S and M estimation. The process begins with S-estimation and then uses the S-estimation estimations as a starting point for M-estimation. Due to the fact that MM estimation combines the other two approaches, it resolves outliers in both the dependent and independent variables. The "MM" in the acronym alludes to the fact that the final estimates are calculated using multiple M-estimation procedures. As with the M-estimation situation, estimates are found using iteratively reweighted least squares (IRLS) (Susanti and Pratiwi, 2014).

\section{Results}

Table 1. Results from M-estimation

Table 1, Table 2, and Table 3 reports the results of M-estimation, S-estimation, and MMestimation, respectively. The results show that all the variables are positive and significant. The $\mathrm{p}-$ values associated with each variable are less than 0.05 . This implies that all the variables has positive and significant impact on cloud migration in healthcare centers.

Dependent Variable: ADOPTION

Method: Robust Least Squares

Sample: 1280

Included observations: 280

Method: M-estimation

$M$ settings: weight=Bisquare, tuning $=4.685$, scale $=$ MAD (median centered)

Huber Type I Standard Errors \& Covariance

\begin{tabular}{ccccc}
\hline \hline Variable & Coefficient & Std. Error & z-Statistic & Prob. \\
\hline \hline COMPATIBILITY & 0.970535 & 0.065203 & 14.88473 & 0.0000 \\
COMPETITIVE & 0.991552 & 0.065280 & 15.18925 & 0.0000 \\
COMPLEXITY & 1.048950 & 0.061825 & 16.96643 & 0.0000 \\
MANAGEMENT & 1.045362 & 0.063994 & 16.33524 & 0.0000 \\
COSTS & 1.000524 & 0.062152 & 16.09793 & 0.0000 \\
SECURITY & 0.950840 & 0.062434 & 15.22941 & 0.0000 \\
TECH & 0.935938 & 0.063483 & 14.74311 & 0.0000 \\
VENDOR & 0.999242 & 0.065009 & 15.37078 & 0.0000 \\
C & 0.542563 & 0.092945 & 5.837439 & 0.0000 \\
\hline \hline & Robust Statistics & $=$ & \\
\hline \hline
\end{tabular}




\begin{tabular}{lllll} 
& R-squared & 0.795481 & Adjusted R-squared & 0.789444 \\
& Rw-squared & 0.899088 & Adjust Rw-squared & 0.899088 \\
Akaike info criterion & 193.1913 & Schwarz criterion & 232.0470 \\
& Deviance & 20.55736 & Scale & 0.336701 \\
& Rn-squared statistic & 1956.286 & Prob(Rn-squared stat.) & 0.000000 \\
\cline { 3 - 5 } Page | 17 & & Non-robust Statistics & \\
\cline { 3 - 5 } & & & & \\
& Mean dependent var & 4.548896 & S.D. dependent var & 0.860785 \\
& S.E. of regression & 0.283765 & Sum squared resid & 21.82161 \\
\hline \hline
\end{tabular}

Table 2. Results from S-estimation

Dependent Variable: ADOPTION

Method: Robust Least Squares

Sample: 1280

Included observations: 280

Method: S-estimation

S settings: tuning $=1.547645$, breakdown $=0.5$, trials $=200$, subsmpl $=9$, refine $=2$, compare $=5$

Random number generator: $r n g=k n$, seed 1299541099

Huber Type I Standard Errors \& Covariance

\begin{tabular}{ccccc}
\hline \hline Variable & Coefficient & Std. Error & z-Statistic & Prob. \\
\hline \hline COMPATIBILITY & 0.759355 & 0.167799 & 4.525390 & 0.0000 \\
COMPETITIVE & 0.928934 & 0.167996 & 5.529518 & 0.0000 \\
COMPLEXITY & 1.160154 & 0.159105 & 7.291763 & 0.0000 \\
MANAGEMENT & 1.087206 & 0.164687 & 6.601639 & 0.0000 \\
COSTS & 0.884754 & 0.159947 & 5.531544 & 0.0000 \\
SECURITY & 0.795254 & 0.160673 & 4.949516 & 0.0000 \\
TECH & 0.749049 & 0.163372 & 4.584937 & 0.0000 \\
VENDOR & 0.784044 & 0.167299 & 4.686477 & 0.0000 \\
C & 0.930422 & 0.239192 & 3.889856 & 0.0001 \\
\hline \hline & Robust Statistics & & \\
\hline \hline R-squared & 0.628453 & Adjusted R-squared & 0.617485 \\
Scale $\quad$ D.330380 & Deviance & 0.109151 \\
Rn-squared statistic & 246.9124 & Prob(Rn-squared stat.) & 0.000000 \\
\hline \hline & Non-robust Statistics & \\
\hline \hline Mean dependent var & 4.548896 & S.D. dependent var & 0.860785 \\
S.E. of regression & 0.308930 & Sum squared resid & 25.86360 \\
\hline \hline
\end{tabular}

Table 1. Results from MM-estimation

Dependent Variable: ADOPTION

Method: Robust Least Squares

Sample: 1280

Included observations: 280 
Method: MM-estimation

S settings: tuning $=1.547645$, breakdown $=0.5$, trials $=200$, subsmpl $=9$, refine $=2$, compare $=5$

$M$ settings: weight=Bisquare, tuning $=4.684$

Random number generator: $\mathrm{rng}=\mathrm{kn}$, seed $=1299541099$

Huber Type I Standard Errors \& Covariance

Page | 18

\begin{tabular}{ccccc}
\hline \hline Variable & Coefficient & Std. Error & z-Statistic & Prob. \\
\hline \hline COMPATIBILITY & 0.970165 & 0.065429 & 14.82777 & 0.0000 \\
COMPETITIVE & 0.991556 & 0.065506 & 15.13696 & 0.0000 \\
COMPLEXITY & 1.049281 & 0.062039 & 16.91329 & 0.0000 \\
MANAGEMENT & 1.045451 & 0.064216 & 16.28031 & 0.0000 \\
COSTS & 1.000320 & 0.062367 & 16.03917 & 0.0000 \\
SECURITY & 0.950688 & 0.062650 & 15.17448 & 0.0000 \\
TECH & 0.935646 & 0.063703 & 14.68770 & 0.0000 \\
VENDOR & 0.999076 & 0.065234 & 15.31524 & 0.0000 \\
C & 0.542940 & 0.093267 & 5.821357 & 0.0000 \\
\hline \hline
\end{tabular}

Robust Statistics

\begin{tabular}{|c|c|c|c|}
\hline R-squared & 0.791705 & Adjusted R-squared & 0.785556 \\
\hline Rw-squared & 0.899274 & Adjust Rw-squared & 0.899274 \\
\hline Akaike info criterion & 200.1956 & Schwarz criterion & 238.6105 \\
\hline Deviance & 20.50924 & Scale & 0.330380 \\
\hline Rn-squared statistic & 1942.501 & Prob(Rn-squared stat.) & 0.000000 \\
\hline \multicolumn{4}{|c|}{ Non-robust Statistics } \\
\hline Mean dependent var & 4.548896 & S.D. dependent var & 0.860785 \\
\hline S.E. of regression & 0.283768 & Sum squared resid & 21.82202 \\
\hline
\end{tabular}

Figure 1. Residuals, Actuals, and Fitted values.

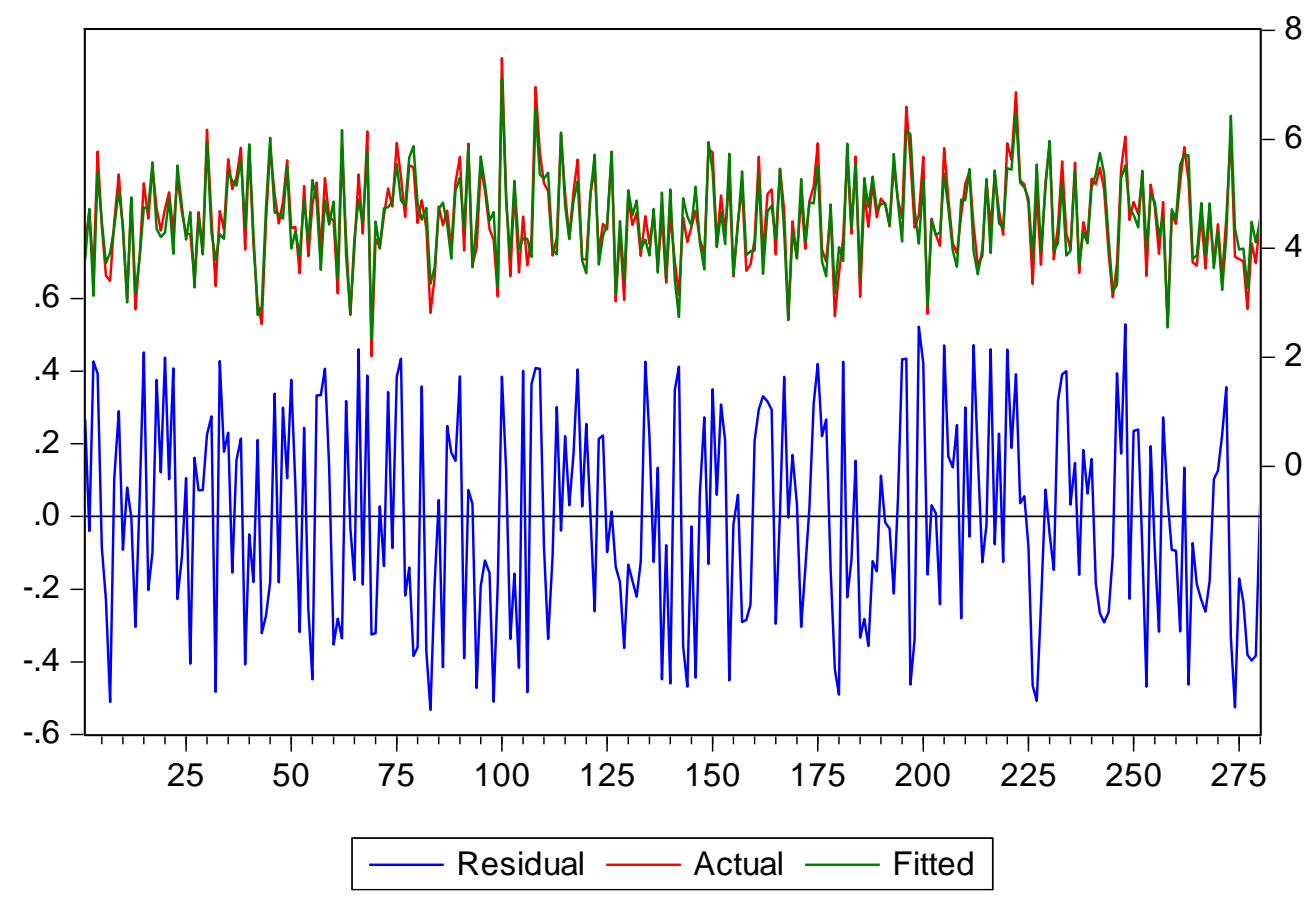


Table 4. Hypothesis decisions after estimation

\begin{tabular}{|l|l|}
\hline Hypotheses & Decisions \\
\hline H1 & Supported \\
\hline H2 & Supported \\
\hline H3 & Supported \\
\hline H4 & Supported \\
\hline H5 & Supported \\
\hline H6 & Supported \\
\hline H7 & Supported \\
\hline H8 & Supported \\
\hline H9 & Supported \\
\hline
\end{tabular}

\section{Conclusion}

Consumers expect their healthcare experience to be the same seamless, digital experience that they get from companies like Amazon. They might not be aware of what is happening behind of the scenes of a health system to optimize their experience. However, consumers will soon demand experiences such as predictive care, accurate staffing, lower medication errors, easy and accurate access to their medical health records, remote-monitored IoT medical devices and more, that they can only get if hospitals support those applications from the cloud.

As Cloud technology progresses exponentially, there is a vast amount of potential for Cloud computing in the healthcare industry. Quality healthcare has acquired the status of a human's fundamental right. Also, this is a costly and highly complicated subject. Even the world's developed economies find it difficult to cope with the sheer scale of healthcare needs. However, channelizing cloud computing power into the healthcare system can surely make tangible progress useful in quality and affordable healthcare for all, rather than a few privileged ones. The world is moving fast and healthcare organizations that digitally transform their businesses and modernize their operations will lead the pack and outpace their competition.

\section{References}

Abiy, R. et al. (2018) 'A Comparison of Electronic Medical Record Data to Paper Records in Antiretroviral Therapy Clinic in Ethiopia: What is affecting the Quality of the Data?', Online journal of public health informatics, $10(2)$.

Alharbi, F., Atkins, A. and Stanier, C. (2016) 'Understanding the determinants of Cloud Computing adoption in Saudi healthcare organisations', Complex \& Intelligent Systems, 2(3), pp. 155-171.

Avram, M.-G. (2014) 'Advantages and challenges of adopting cloud computing from an enterprise perspective', Procedia Technology, 12, pp. 529-534. 
Dang, L. M. et al. (2019) 'A survey on internet of things and cloud computing for healthcare', Electronics, 8(7), p. 768.

Doukas, C. and Maglogiannis, I. (2012) 'Bringing IoT and cloud computing towards pervasive healthcare', in 2012 Sixth International Conference on Innovative Mobile and Internet Services in Ubiquitous Computing. IEEE, pp. 922-926.

Page | 20

Faulina, N. (no date) 'Robust Regression M-Estimation, S-Estimation, and MM-Estimation in Multiple Regression'.

Mallmann, G. L. and Maçada, A. C. G. (2018) 'Adoption of cloud computing: a study with public and private hospitals in a developing country', International Journal of Innovation and Technology Management, 15(05), p. 1850044.

ogly Alguliev, R. M. and ogly Alekperov, R. K. (2013) 'Cloud computing: Modern state, problems and prospects', Telecommunications and Radio Engineering, 72(3).

Ogwel, B., Otieno, G. and Odhiambo, G. (2020) 'Cloud Computing Adoption by Public Hospitals in Kenya A Technological, Organisational and Behavioural Perspective’.

Qasem, Y. A. M. et al. (2020) 'A Multi-Analytical Approach to predict the determinants of cloud computing adoption in higher education institutions', Applied Sciences, 10(14), p. 4905.

Rahimi, F., Møller, C. and Hvam, L. (2016) 'Business process management and IT management: The missing integration', International Journal of Information Management, 36(1), pp. 142-154.

Ratnam, K. A. and Dominic, P. D. D. (2014) 'Adoption of cloud computing to enhance the healthcare services in Malaysia', in 2014 International Conference on Computer and Information Sciences (ICCOINS). IEEE, pp. 1-6.

Shimrat, O. (2009) 'Cloud computing and healthcare', San Diego Physician. org, pp. 26-29.

Sulaiman, H. and Magaireah, A. I. (2014) 'Factors affecting the adoption of integrated cloudbased e-health record in healthcare organizations: A case study of Jordan', in Proceedings of the 6th International Conference on Information Technology and Multimedia. IEEE, pp. 102-107.

Sultan, N. (2014) 'Making use of cloud computing for healthcare provision: Opportunities and challenges', International Journal of Information Management, 34(2), pp. 177-184.

Susanti, Y. and Pratiwi, H. (2014) 'M estimation, S estimation, and MM estimation in robust regression', International Journal of Pure and Applied Mathematics, 91(3), pp. 349-360. 